\title{
Effect of Integration of Science and Technology Information on Achievements Transformation
}

\author{
Y.Q. XIA, X.C. WEI, Y.Y. HOU \& H.Q.LV \\ Beijing Municipal Institute of Science and Technology Information, China
}

\begin{abstract}
In recent years, with the rapid development of economy, China's investment in the field of scientific and technological research has gradually increased. Despite the increasing quantity of scientific and technological research achievements year by year, the rate of achievements transformation (AT) in China is still very low. They cannot be transferred spontaneously into practical production and are inseparable from the scientific and technological information (STI) service. The goal of this research is to establish a suitable method to improve services for the government with STI. In this study, effect of integration of science and technology information on achievements transformation is discussed.
\end{abstract}

KEYWORD: Scientific and Technological Information; Integration; Achievements Transformation; China

\section{INTRODUCTION}

To promote the development of science and technology is an inexhaustible force for the growth of national economy and social science. The transformation of research achievements is an important way to boost scientific and technological development. However, the achievements of scientific and technological cannot spontaneously transfer into practical productive forces. They are inseparable from the science and Technology Information Service. Therefore, we need to better utilize scientific and technological information with an effective method.

In this paper, in order to obtain the basic data to establish a suitable method for technology information service for government, the China's status of AT, and effect of integration of STI on AT were discussed.

\section{STATUS OF CHINA'S AT}

In recent years, with the rapid development of economy, China's investment in the field of scientific and technological research has gradually increased as well (Fig 1).

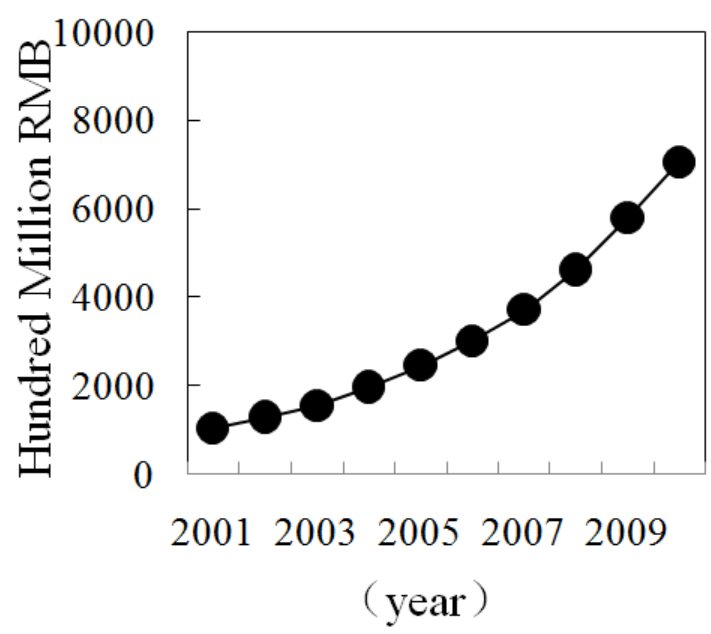

Fig 1. The change of investment of scientific research in China during 2001 to 2010 .

The increase of investment lead to quantitive increase of scientific and technological research achievements made by the universities and research institutions in China (Fig. 2 and 3). Despite the increasing quantity of scientific and technological research achievements year by year, the transformation rate of scientific and technological achievements in China is still very low. It was reported by a study that the transformation rate of science and technology achievement in China is less than $10 \%$.

Generally, scientific and technological achievements cannot be transferred spontaneously into a practical production. They are inseparable from scientific and technological information 
service. As such, effectively connecting the market and technology with STI becomes inevitable in promoting the transformation rate of scientific and technological achievements. However, at the age of big data, we are facing a vast sea of data. Therefore, we have to understand the characteristic of STI and integrate STI before we using it in an effective way.

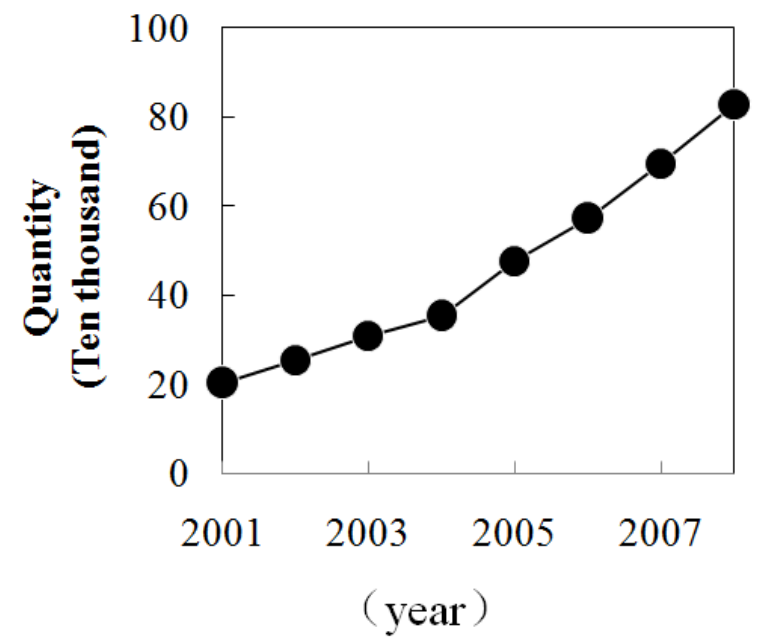

Fig 2. The number change of the China for patent application during 2001 to 2008 .

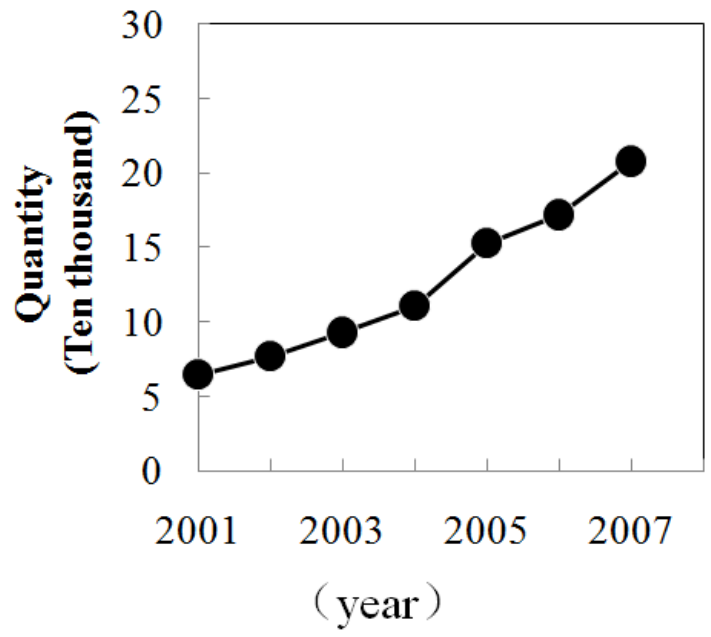

Fig3. The number change of the China for patent grant during 2001 to 2008.

\section{STI INTEGRATION}

To integrate STI, we need to analysis the characteristic of STI's elements and the according classification .

\subsection{Characteristic of STI's factors}

STI can be searched from internet, database and other ways. The content and structure of STI are very complex. From a wider view, personnel, financial, material, technology information all should be considered as a part of STI, and all the STI includes factors of information, knowledge, organization, system, policy, intermediary service, culture of innovation, funds personnel, technology, and so on. Characteristics of these main factors for improving AT are as follows:

(1) Information: Determine the efficiency of STI resource allocation. The scope of application is all AT aspects.

(2) Knowledge: Source of scientific and technological innovation. Play a proactive role in STI resource allocation for achievements transformation.

(3) Organization: Scale and intension for a group of people who work together.

(4) System: Provide protection for AT in specific issues, different from scattered, small-scale AT activities.

(5) Policy: Macro protection. Apply to new government decisions of the on achievements transformation.

(6) Intermediary service: An effective link in AT activities, usually provided by a variety of small organizations with different content of achievements transformation.

(7) Funds: The most critical factor of AT, to provide financial support.

(8) Personnel: Initiator, the carrier of positive factors, knowledge and technology, integrate all the resources for achievements transformation.

(9) Technology: "Soul" of research achievements transformation.

The above-mentioned characteristics show the significance of STI's factors. However, STI's factors keep an important a role in achievement transformation in such a long term process that we need to adjust STI's function according to specific condition.

\subsection{Classification}

In light with analysis above, all the STI's factors can also be divided into two types in terms of morphology and number measurability, which sometimes are also called as soft science and hard science.

Soft science includes technology information, knowledge, organization, systems, policies, services culture.

Hard science includes funds, personnel and technology for characteristic of is accumulated from both knowledge and technological innovation .

Integration of STI can play a very important role in the development of national economy and social sciences. Effective integration of STI resources can support researchers to improve the quality of their work. This is a good way for application because many of innovation elements such as expert wisdom, science and technology information resources and computer technology can all be combined together. 


\section{EFFECT OF INTEGRATION OF STI ON AT}

\subsection{Foundation for effective analysis}

The promotion of scientific and technological achievement transfer rate cannot survive without industry development and technology innovation. AT are based upon the analysis of STI processing. In recent years, many analysis methods for scientific and technical information have been developed around the world. They can be divided into the following five categories: quantitative analysis vs qualitative analysis method, longitudinal contrast vs horizontal contrast, macro analysis vs micro analysis, patent search vs literature analysis, study design vs decision-making application method. However, the use of these analysis methods builds on the foundation of integration of STI.

\subsection{Guiding role}

Integration of STI can play a guiding role for AT from a long - term point view in industry development. AT needs to be carried with in line with market demands which can help to determine the total amount, varieties of a product. A clear aspect of AT can make the products have a good market, then the AT can obtain the economic benefits of good. Compared with other developing countries, China's integration of STI is still poor. STI resources integration is a long term process that is accumulated from both knowledge and technological innovation.

Scientific and technological information resources play a very important role in the development of national economy and social sciences. China is currently constructing an innovative country of science and technology. In order to reach this goal, the government has to evaluate various innovative capabilities such as system, policy and working practice effect in regular time. This is not an easy job, and first we need to think how to integrate STI and provide STI to the government for promoting AT. This is also an important duty for any scientific and technological information service organization.

\section{CONCLUSION}

The transformation of scientific and technological achievements is a complex systematic process, which requires governments, universities, research institutes, enterprises, market and capital play their own role in the development of scientific and technological system. Therefore, it is very necessary to integrate STI and utilize STI to effectively connect all these elements together.

\section{REFERENCES}

[1] SMEDLUND A. The roles of intermediaries in a regional knowledge system. Journal of Intellectual Capital, 2006, 7: 204-220.

[2] Fernandes, L. R.; Antunes, A. S. Strategic information on medicinal plants obtained from online databases. Agro Food Industry Hi-Tech, 2002, 13, 6: 13-18.

[3] SM ITS R, KUHLMANN S. The rise of systemic instruments in innovation policy. International Journal of Foresight and Innovation Policy, 2004, 1:4-30.

[4] HEIDRUN C HOPPE, EMRE OZDENOREN. Intermediation in innovation. International Journal of Industrial Organization, 2005, 23:483-503.

[5] JEREMY HOWELLS. Intermediation and the role of intermediaries in innovation. Research Policy, 2006, 35:715-728. 